\title{
Sensitivity Study of Geomechanical Effects on Reservoir Simulation
}

\author{
L.C. Pereira Petrobras - Petróleo Brasileiro S.A., Brazil
}

L.J.N. Guimarães University of Pernambuco, Brazil

F.O.L. Falcão Petrobras - Petróleo Brasileiro S.A., Brazil

\begin{abstract}
Classical reservoir simulation considers rock compressibility as the only geomechanical parameter influencing field production. It is assumed to be constant or vary only with oil phase pressure. However, a conventional simulator still cannot explain some phenomena occurring during production, such as compaction, casing damage, wellbore stability, pore collapse, fault reactivation etc. In order to consider the geomechanical influence on the reservoir behaviour, it is necessary to use a coupled model: constitutive laws, mechanical properties of the reservoir and surrounding rocks, state of stress etc. On the other hand, the uncertainties inherent to the mechanical properties are enormous. To properly characterise a rock, lab tests are necessary, but cores are rarely available for destructive tests. Another solution is to use correlations between seismic response and rock properties. But, how reasonable and accurate are such correlations? This work presents a methodology of geomechanical modelling considering a sensitivity study of geomechanical parameters. Simulations with the commercial software STARS® were carried out, which identified the main geomechanical parameters relevant in flow simulation through a sensitivity analysis, based on a sugar cube model. Based on these results, the impact of geomechanics on reservoir simulation is illustrated by comparing permeability, porosity, oil and water production values generated by conventional and coupled simulations.
\end{abstract}

\section{Introduction}

Reservoir simulation has technologically developed significantly in the last decades. Along with the fast evolution of computation capacity, recent developments of reservoir simulators have helped increase its applications: vertical flow in wells and fluid flow in porous media integration, optimisation and uncertainty analyses, integration with surface facilities and geomechanical modelling. The focus of the present work is on geomechanical and reservoir simulation coupling, the characteristics of such an approach and how this approach impacts on final results.

In conventional reservoir simulation the only geomechanical parameter involved is rock compressibility. This parameter is not sufficient to represent rock behaviour in response to stress variations. Further, the variation of pore volume due to overburden loading cannot be considered by only using the compressibility value. For example, the porosity reduction in depleted areas may be sufficiently high to cause pore collapse and significantly modify the reservoir behaviour thereafter. Thus, this work intends to study the reservoir compaction phenomenon by solving the fluid flow coupled to the geomechanical model. In addition, a comparison of results between conventional and coupled simulation is presented. We also raise issues and provide results that clarify the importance of geomechanics in reservoir simulation. With this purpose in mind, a case of reservoir compaction with very representative data will be discussed.

\section{Flow with geomechanical coupling - mathematical formulation}

The purpose of this section is to review some basic equations related to this study rather than give details of flow equations used in commercial simulators.

\subsection{Mass conservation}

The simulation of reservoir behaviour is carried out based on mass conservation of fluids and the species contained in it. Considering the geomechanical coupling, the reservoir rock is deformable and the variation 
of its porosity is calculated in the solid phase mass conservation equation. In this section we derive the conservation equation of a generic physical quantity that shall be applied in the following sections.

Let $\psi$ be a physical quantity per unit mass, which may be a scalar, vector or a tensor, at any point belonging to volume $V$ at time $t ; \Omega$ is the supply of $\psi$ per unit mass; $\Gamma . n$ is the flux of $\psi$ per unit area in $V$. Thus, the general conservation equation for a single phase within the volume $V$ is written as:

$$
\frac{d}{d t} \int_{V} \rho \psi d V=\int_{V} \rho \Omega d V-\int_{S} \Gamma . n d S
$$

Applying the divergence theorem to the last term on the right side of Equation (1) gives:

$$
\int_{S} \Gamma \cdot n d S=\int_{V} \nabla \cdot \Gamma d V
$$

Using Reynolds transport theorem (Lin and Segel, 1974) and using Equation (2), the term on the left side of Equation (1) gives:

$$
\frac{\partial(\rho \psi)}{\partial t}+v \cdot \nabla(\rho \psi)+(\rho \psi) \nabla \cdot v+\nabla \cdot \Gamma-\rho \Omega=0
$$

where $v$ is the velocity vector of the material. Equation (3) is applicable at any point of the porous medium.

\subsubsection{Mass conservation for fluids}

In this section the mass conservation law is applied to a one phase saturated medium. Porosity $\phi$ is defined as the ratio between pore volume and bulk volume. Considering $\psi=\phi ; \Omega=0, \nabla . \Gamma=-Q ; v=v_{f}$ and $\rho=\rho_{f}$, let $Q$ be the fluid flow; $v_{f}$ the fluid velocity and $\rho_{f}$ the fluid density. Replacing these terms in Equation (3):

$$
\frac{\partial\left(\phi \rho_{f}\right)}{\partial t}+\nabla\left(\phi \rho_{f} v_{f}\right)-Q=0
$$

\subsubsection{Mass conservation for solids}

The mass conservation for the solid rock can be similarly obtained setting $\psi=1-\phi ; \Omega=0 ; \Delta . \Gamma=0 ; v=v_{r}$ and $\rho=\rho_{f}$, let $v_{r}$ be the rock velocity and $\rho_{r}$ the solid density. Replacing these terms in Equation (3):

$$
\frac{\partial\left[(1-\phi) \rho_{r}\right]}{\partial t}+\nabla\left[\rho_{r}(1-\phi) v_{r}\right]=0
$$

where $v_{r}$ is the unknown factor for the geomechanical problem.

\subsection{Darcy's law}

In addition to the mass balance equation, a relationship or law between the fluid velocity and the pressure gradient is required. Darcy's law governs the fluid transportation within the porous medium. This law relates the apparent fluid velocity to pressure gradients through the equation:

$$
v=-\frac{k}{\mu} \cdot\left(\nabla p-\rho_{f} b\right)
$$

Where $k$ is the fluid permeability tensor; $p$ is the pore pressure; $\mu$ is the fluid viscosity and $b$ the body force per unit mass.

For a deformable porous medium, the velocity in Darcy's equation (also called surface velocity, which is the fluid velocity in relation to the solid phase) can be related to fluid velocity $v_{f}$ and solid rock velocity $v_{r}$ (Hart and John, 1986). $v_{r}$ is the solid phase velocity in relation to the reference configuration (intact rock). 


$$
v_{f}=\left(\frac{v}{\phi}+v_{r}\right)
$$

Applying Equations (6) and (7) to Equation (4):

$$
\frac{D}{D t}\left(\phi \rho_{f}\right)-\nabla\left(\rho_{f} \frac{k}{\mu} \cdot\left(\nabla p-\rho_{f} b\right)\right)-\phi \rho_{f} \frac{D \varepsilon_{v}}{D t}=Q_{f}
$$

Equation (8) shows the interaction between fluid and rock through pore pressure and volumetric strain $\mathcal{E}_{v}$. In a conventional simulator, the last term on the left hand side of Equation (8) is ignored due to the consideration that the bulk volume is not varying with time. Without coupling, the true porosity is replaced by reservoir porosity:

$$
\phi^{*}=\frac{V_{p}}{V_{b}^{0}}
$$

By definition, the reservoir porosity is the ratio between current pore volume and initial bulk volume, whereas true porosity is a ratio between the current pore volume and current bulk volume. In a conventional reservoir simulator, the porosity is restricted to be pressure and temperature dependent, only because stresses are not part of the solution.

\section{Two-way coupling}

Fluid flow and formation deformation (geomechanics) are coupled in a sequential manner, that is, the two calculations are done alternately, while passing information back and forth. The fluid flow and temperature calculation updates the pressures and temperatures over a specific time-step. The geomechanics module updates the formation deformation in response to the new pressure and temperature. To complete the loop, the geomechanics module sends the new deformation information back to the fluid flow calculation to be used in the next time-step. It is clear that information flows from fluid flow to geomechanics via pressure and temperature. However, it is not obvious how information goes the other way back.

The fluid flow module calculates porosity as a function of pressure and temperature, considering that pore volume and hence mass is conserved between time steps. Here, conserved means that the porosity at the beginning of a time step is equal to the porosity at the end of previous time step, at that particular pressure and temperature. When the porosity function $\varphi(\mathrm{p}, \mathrm{T})$ itself does not change with time, mass conservation across time steps is ensured.

However, the porosity function can vary between time steps and still conserve mass. Let $\mathrm{p}^{\mathrm{n}}$ and $\mathrm{T}^{\mathrm{n}}$ be the solution for a grid block for time step " $n$ " that calculates porosity as a function of $\varphi^{n}(p, T)$. The next time step " $\mathrm{n}+1$ " starts with $\mathrm{p}=\mathrm{p}^{\mathrm{n}}$ and $\mathrm{T}=\mathrm{T}^{\mathrm{n}}$ but has a different porosity function $\varphi^{\mathrm{n}+1}(\mathrm{p}, \mathrm{T})$. Porosity and hence mass will be conserved between these two time steps if $\varphi^{n}\left(p^{n}, T^{n}\right)=\varphi^{n+1}\left(p^{n}, T^{n}\right)$. However, $\varphi^{n}$ and $\varphi^{n+1}$ may have different derivatives with respect to dependent variables $p$ and $T$ at $p=p^{n}$ and $T=T^{n}$.

The geomechanical deformation response is expressed in the fluid flow calculation by changing parameters in the porosity function. These parameters are kept constant during time step convergence but are updated between time steps such that porosity and hence mass is conserved. The deformation response is accounted on a block-by-block basis since each grid block has its own set of parameters that are porosity dependent.

The coupling method used in the case study presented in this work considers the porosity variation as pressure, temperature and total average stress dependent, following (Stars Technical Guide, 2001):

$$
\phi^{n+1}=\phi^{n}+\left(c_{o}+c_{2} a_{1}\right)\left(p-p^{n}\right)+\left(c_{1}+c_{2} a_{2}\right)\left(T-T^{n}\right)
$$

where:

$$
c_{o}=\frac{1}{V_{b}^{0}}\left(\frac{d V_{p}}{d p}+V_{b} \alpha c_{b} \frac{d \sigma_{m}}{d p}-V_{p} \beta \frac{d T}{d p}\right)
$$




$$
\begin{gathered}
c_{1}=\frac{V_{p}}{V_{b}^{0}} \beta \\
c_{2}=-\frac{V_{b}}{V_{b}^{0}} \alpha c_{b} \\
a_{1}=\text { factor }\left(\frac{2}{9} \frac{E}{(1-v)} \alpha c_{b}\right) \\
a_{2}=\text { factor }\left(\frac{2}{9} \frac{E}{(1-v)} \beta\right)
\end{gathered}
$$

$c_{b}$ is the bulk compressibility, $E$ the Young's modulus, $V_{b}{ }^{0}$ the initial bulk volume, $V_{b}$ the bulk volume, $\alpha$ the Biot number, $v$ Poisson's ratio, $V_{p}$ the pore volume, $\sigma_{m}$ mean total stress, $\beta$ the volumetric thermal expansion coefficient of the formation and factor is a boundary condition number.

\section{Sensitivity analyses on geomechanical parameters}

This section presents the concepts of experimental design and response surface. Next, the sugar cube model is described, including its physical characteristics, geometry and simulation control. Further, we present the parameters involved, a case study is given and finally the results with relevant discussions are provided.

\subsection{Experimental design}

The experimental design is a statistical technique widely used in other exact and human sciences areas, such as chemistry and biology. It is based on the collection of the largest amount of information using the least number of experiments possible, simultaneously varying all uncertainty parameters. In reservoir engineering, the physical process is replaced by flow simulation, making it a numerical experiment. Therefore, the experimental design corresponds to a specific group of reservoir flow simulations that covers the domain of uncertainties in an optimised form.

The experimental design has several advantages: it decreases the number of simulations; it studies the factors that directly influence the response; it considers interaction between factors; it produces optimal levels; and it provides accurate results.

The objective of this paper is to use experimental design to carry out the analysis of parameters that have a major influence on cumulative oil production, through the variation of geomechanical parameters, such as elasticity modulus, Poisson ratio, Biot's coefficient, friction angle, cohesion and cap position. This methodology, based on sensitivity analyses, will show the influence of geomechanical modelling on flow simulation. Its impact will be measured by comparing the cumulative field oil production predicted in both ways; considering the rock compressibility as the only geomechanical parameter, or coupled to a geomechanical module.

\subsection{Response surface}

The response surface methodology is essentially a set of statistical techniques with the purpose of providing a greater knowledge of certain phenomena. The surface is built considering the results of experimental design simulations that attempts to relate responses to quantitative factor levels that influence them. The objectives of relating answers to influence factor are: establish a description of how a response is affected by a number of factors in some interest region; explore the relation among various responses and mandatory extremes; locate and explore the maximum or minimum responses.

Finally, the response surface model is fast to run and may replace the simulator results (which take much more time to be obtained) with reasonable precision, in a certain domain of parameter variation. The model may be used, for example, to find the interest variable values, such as cumulative oil production and oil rate. 


\subsection{Synthetic model}

The synthetic model has been built using software STARS®. The arrangement built represents repetition cells that shall be reflected on axes (X and $\mathrm{Y}$ ) in order to obtain a complete reservoir mesh. The five-spot system was considered and $1 / 4$ modelled.

\subsubsection{Geometry}

This sugar cube model has fifteen cells in $\mathrm{X}$ direction, fifteen cells in $\mathrm{Y}$ direction and eleven cells in $\mathrm{Z}$ direction. The reservoir top is at $8220 \mathrm{~m}$. Wells are vertical and their diameters, both the producer and injector, are $0.216 \mathrm{~m}$. The model geometry is presented in Figure 1.

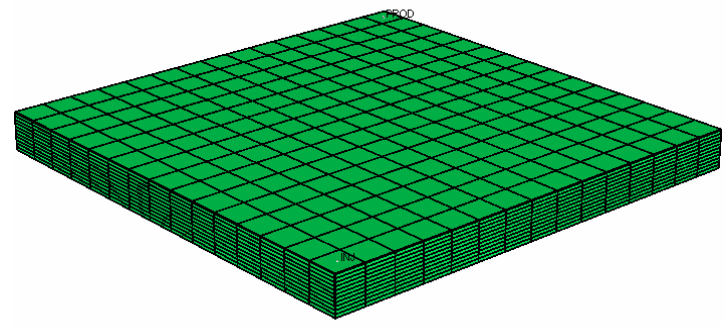

\section{Figure 1 Reservoir geometry}

\subsubsection{Physical characteristics}

The model has constant porosity $(\phi)$ equals to 0.2 . The horizontal permeabilities $\mathrm{K}_{\mathrm{x}}$ and $\mathrm{K}_{\mathrm{y}}$, are equal to $40 \mathrm{mD}$, while the permeability in vertical direction is $4 \mathrm{mD}$. Considering that the bubble point pressure will not be reached during exploitation, the only phases presented are oil and water.

Further model characteristics are: oil viscosity has been defined as $14.38 c P$, the water viscosity equals to $0.4 \mathrm{cP}$ and the initial oil saturation $\left(S_{o i}\right)$ is equal to 0.68 .

\subsubsection{Simulation control}

Pressure maintenance is a common practice in oil reservoir development and presents some advantages like maintaining the reservoir under-saturated, since this avoids the release of associated gas and quickly increase of gas-oil ratio on producer wells. As mentioned previously, this sugar cube model has two vertical wells, one injector and one producer. The numerical simulator well controls are as follows: the wells are completed in all its extension, and are closed in the beginning of the simulation. The first well control of the producer is defined as maximum oil rate of $3000 \mathrm{bbl} / \mathrm{d}\left(5.5210^{-3} \mathrm{~m} / \mathrm{s}\right)$, if this condition is not reached, the second control is minimum bottom hole pressure equal to $1000 \mathrm{psi}(6.89 \mathrm{MPa})$. For the injector well, the first control is the maximum water rate in reservoir conditions of $3000 \mathrm{STB} / \mathrm{d}\left(5.5210^{-3} \mathrm{~m}^{3} / \mathrm{s}\right)$.

The simulation time is 30 years, beginning on 31 December 2008. After the initialisation and pressures stabilisation, the producer well is opened on 30 June 2009. The production starts and the reservoir reach an advanced stage of depletion. On 31 December 2013 the injector well is opened and both wells remain opened until the end of the simulation on 31 December 2039. The reason of such a high depletion is that this provides a better case for observing the geomechanical effects during simulation.

\subsubsection{Parameters in sensitivity analyses}

The parameters ranges have been defined in order to cover the maximum number of reservoirs found on Brazilian basins. The parameters variation that shall be interpreted by COUGAR® to carry out the sensitivity analysis is defined in Table 1. 
Table 1 Geomechanical parameters

\begin{tabular}{ll}
\hline Parameter & Range \\
\hline Biot's coefficient (bio) & $0.8-1.0$ \\
Young's modulus (e) & $8 \mathrm{E} 5-2 \mathrm{E} 6 \mathrm{psi}(5.52-13.8 \mathrm{GPa})$ \\
Friction angle (fricangle) & $30-40\left(^{\circ}\right)$ \\
Poisson's ratio (poisson) & $0.2-0.4$ \\
Cohesion (coe) & $1000-3000 \mathrm{psi}(6.89-20.68 \mathrm{MPa})$ \\
\hline
\end{tabular}

\subsection{Methodology and results}

The following methodology was used:

- Build the reservoir model using STARS®.

- Experimental design using square surface methodology. With parameters defined in Table 1, the experimental design required 27 simulations. Each simulation took about 30 minutes.

- Response surface generation considering cumulative oil production as output parameter. Validate this surface by comparing results with simulator response. Present results as cumulative oil production, water cut and porosity average distribution.

- Critical analyses in cumulative oil production, water cut and porosity average distribution. These results were always compared to conventional simulation (which considers the rock compressibility as the only geomechanical parameter).

\subsubsection{Elastic regime}

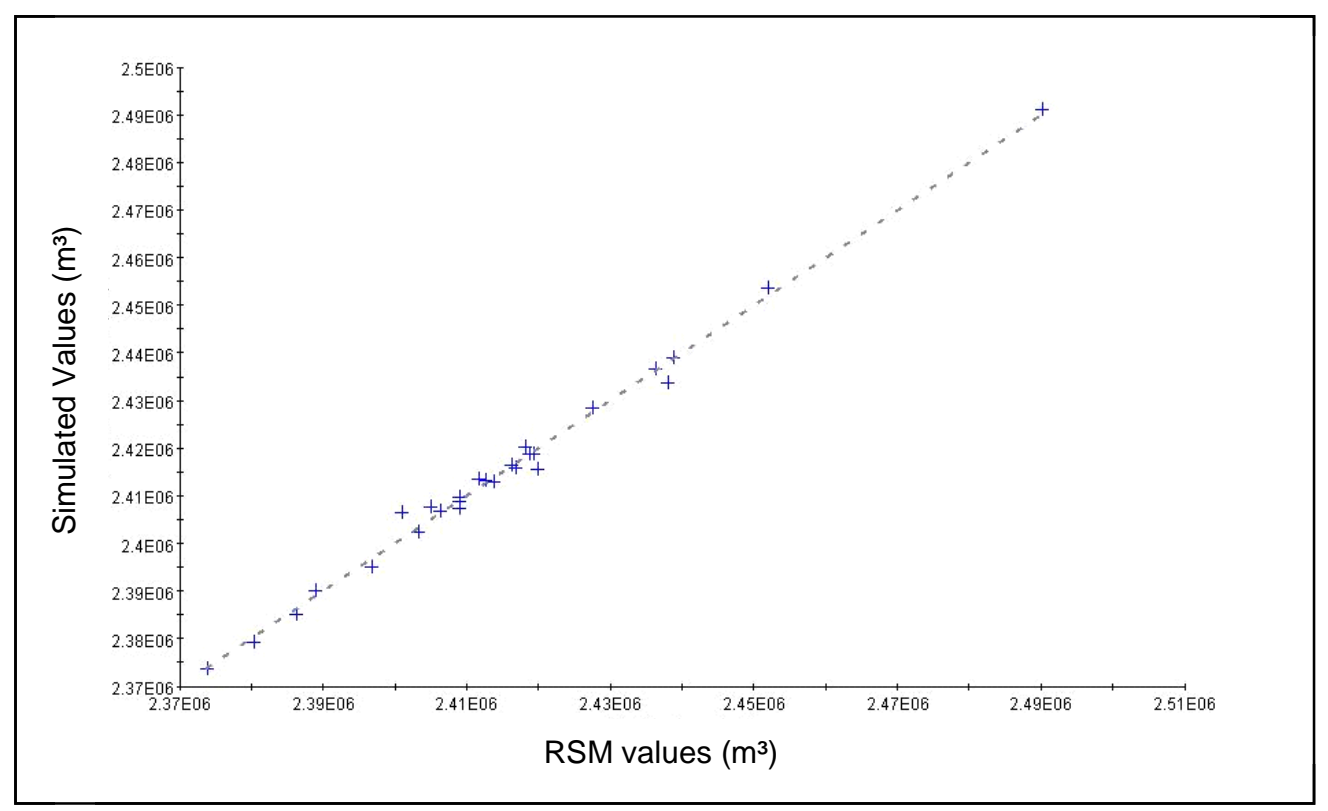

Figure 2 Response surface model $(\mathrm{RSM})$ values $\mathrm{x}$ simulation values 


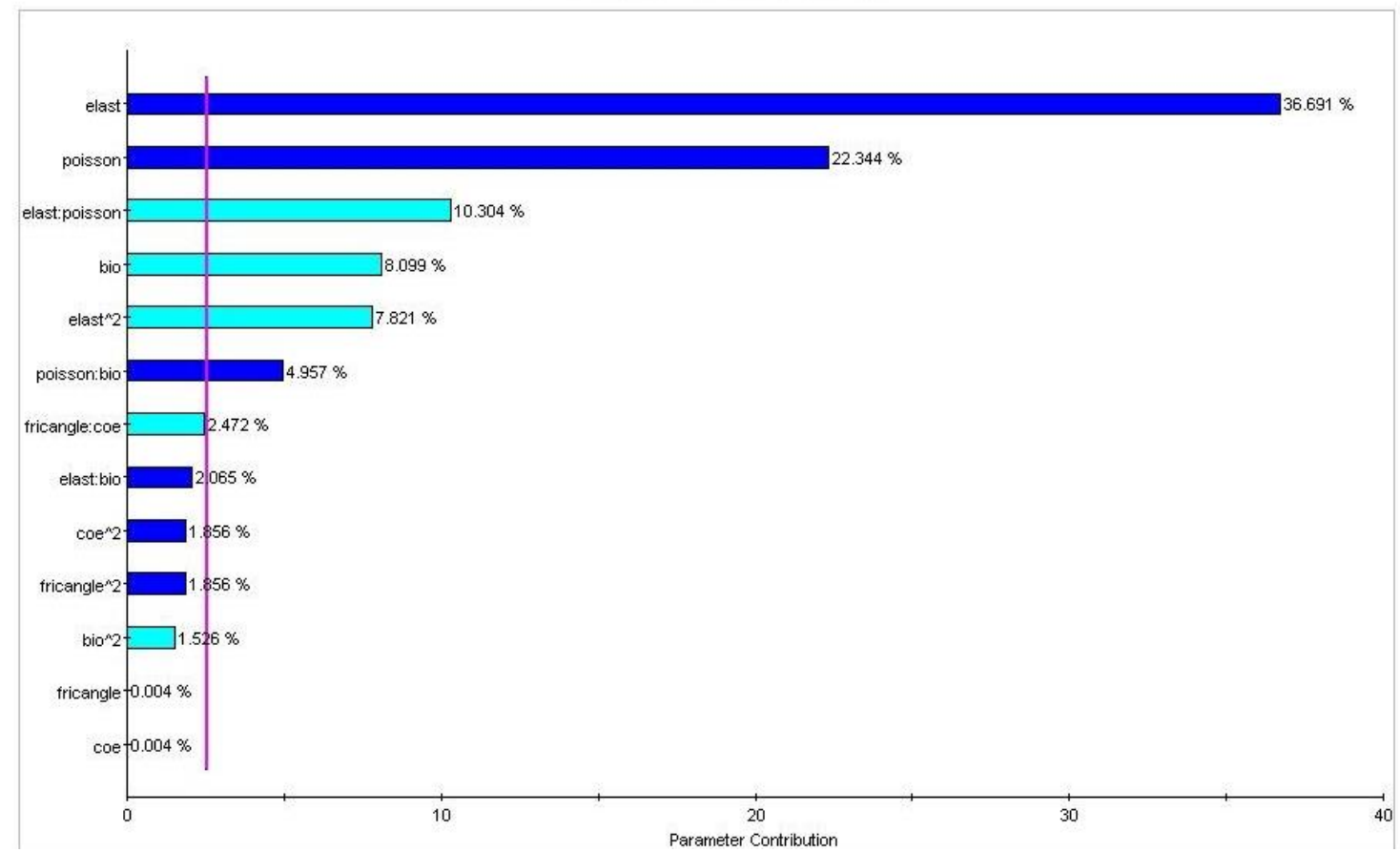

Figure 3 Influence on response surface

\subsubsection{Plastic regime}

Cumulative Oil SC_MELL_PROD_Time_11322.0

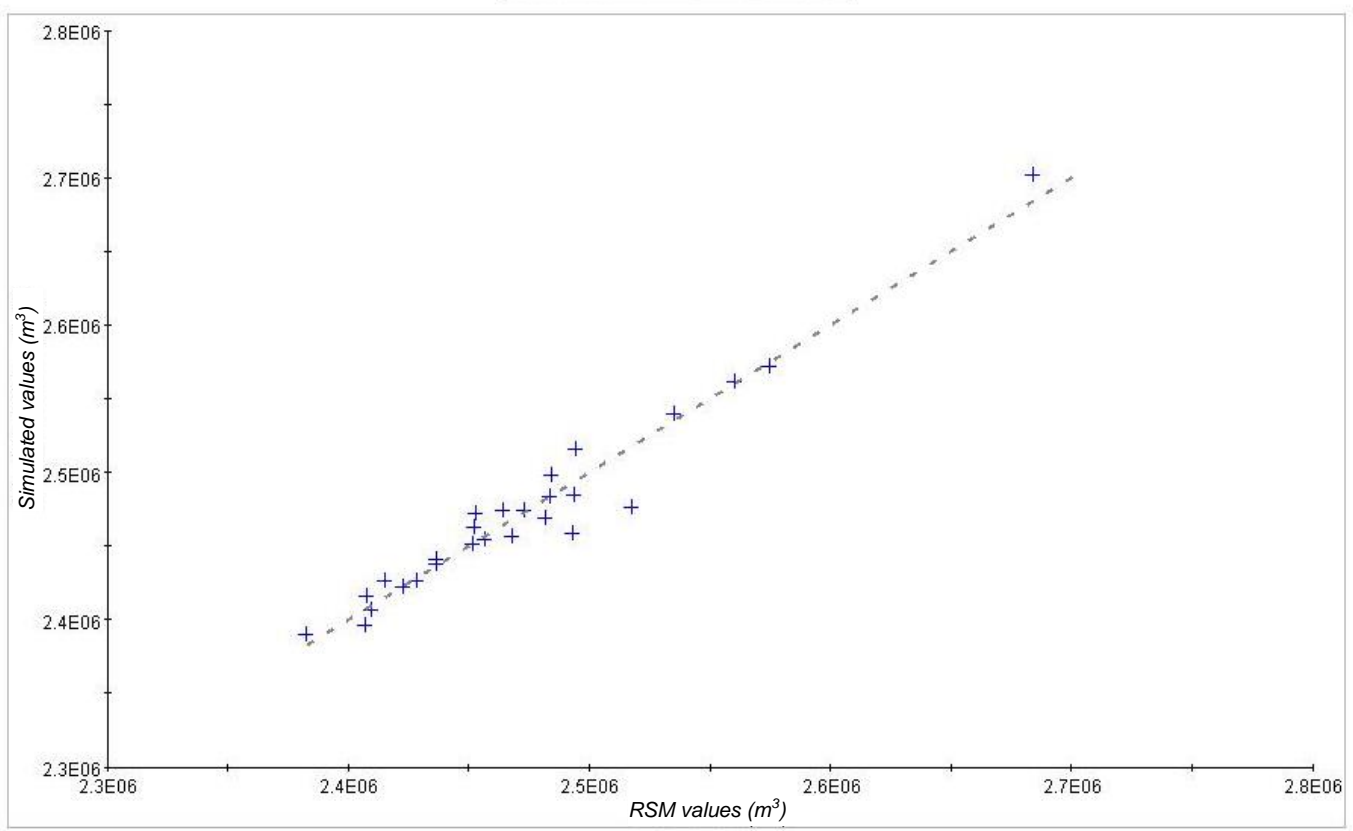

Figure 4 Response surface model (RSM) values $x$ simulation values 


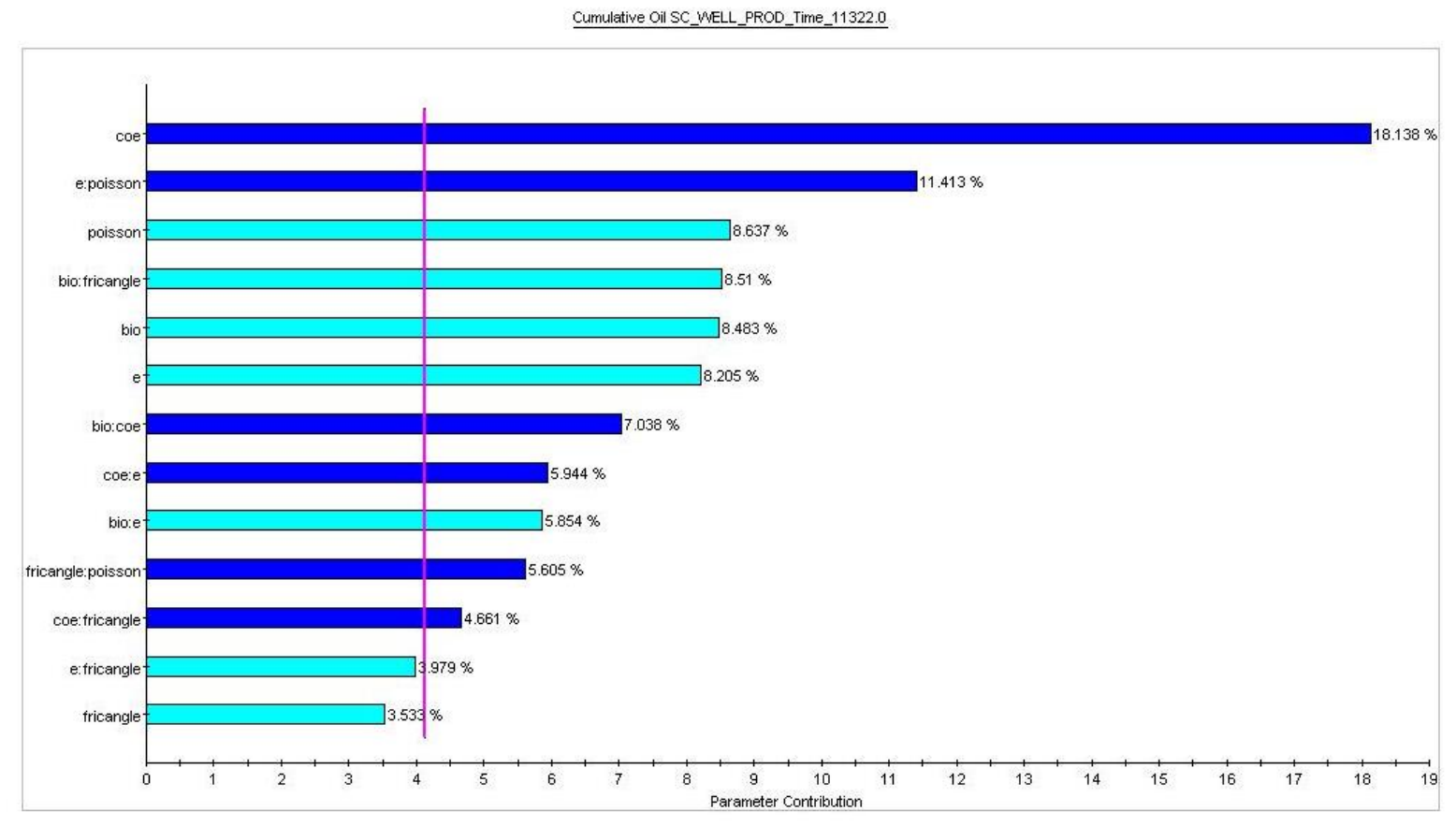

\section{Figure 5 Influence on response surface}

\subsubsection{Results}

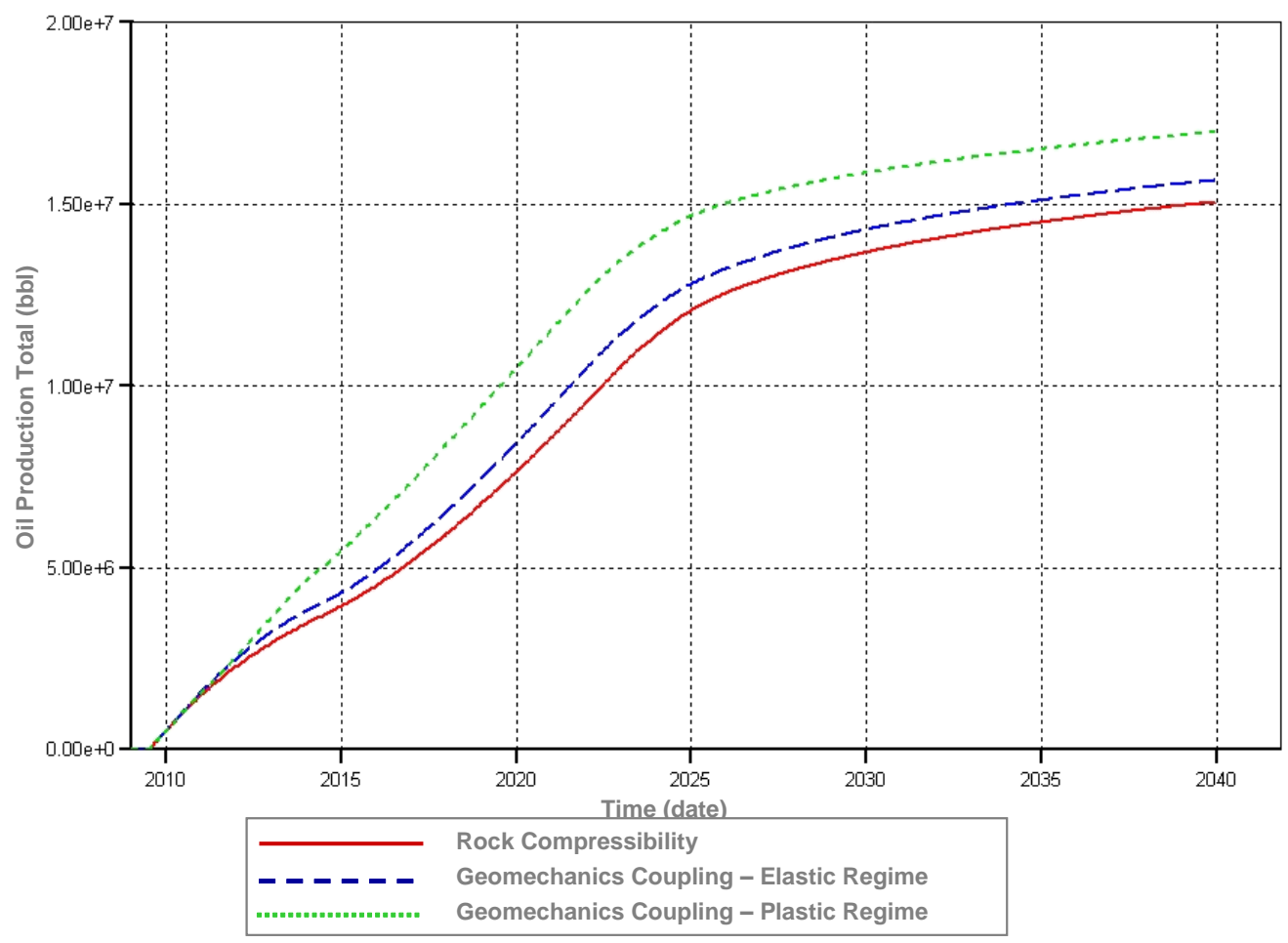

Figure 6 Cumulative oil production (bbl/d) 


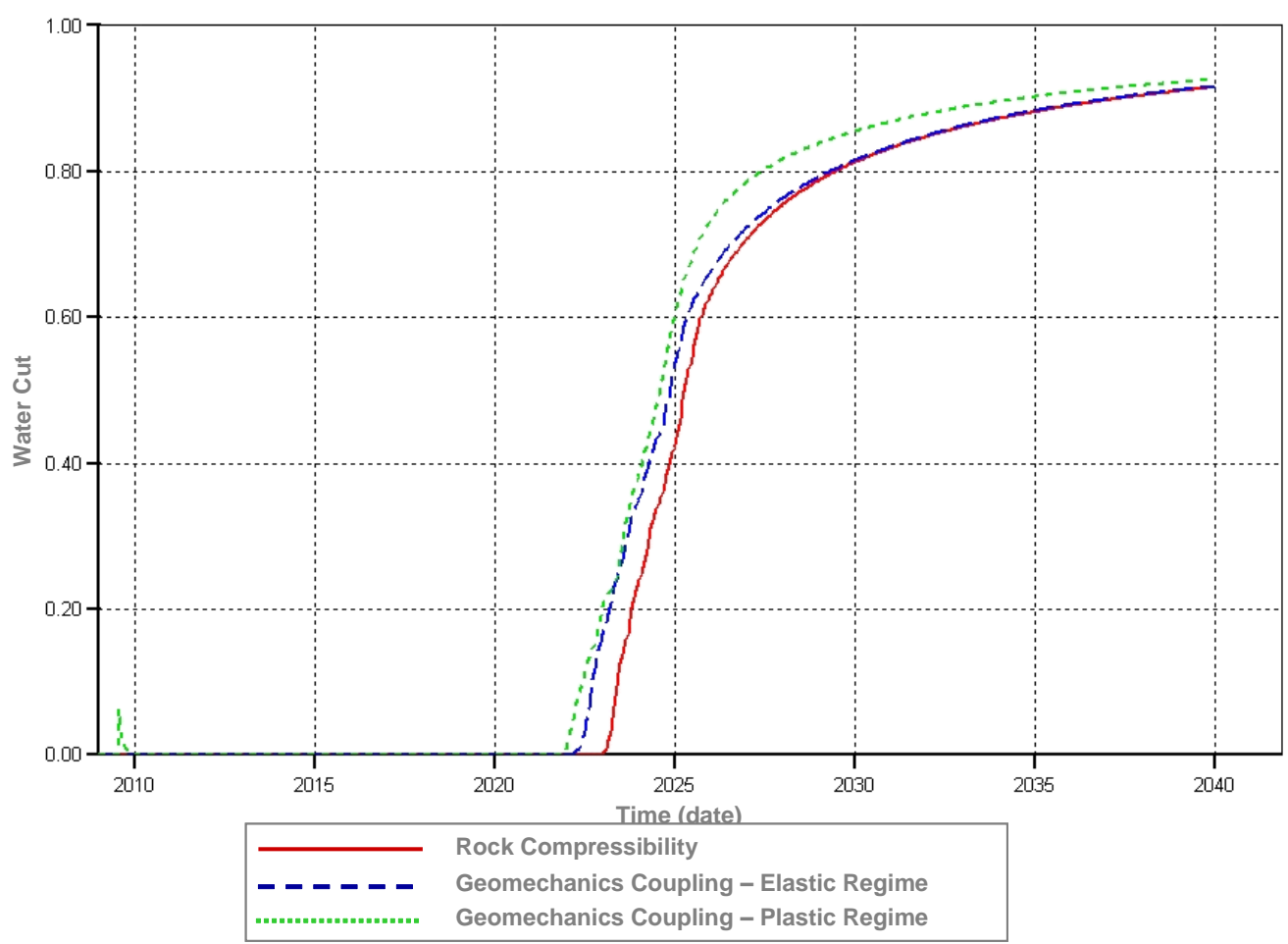

\section{Figure 7 Water cut}

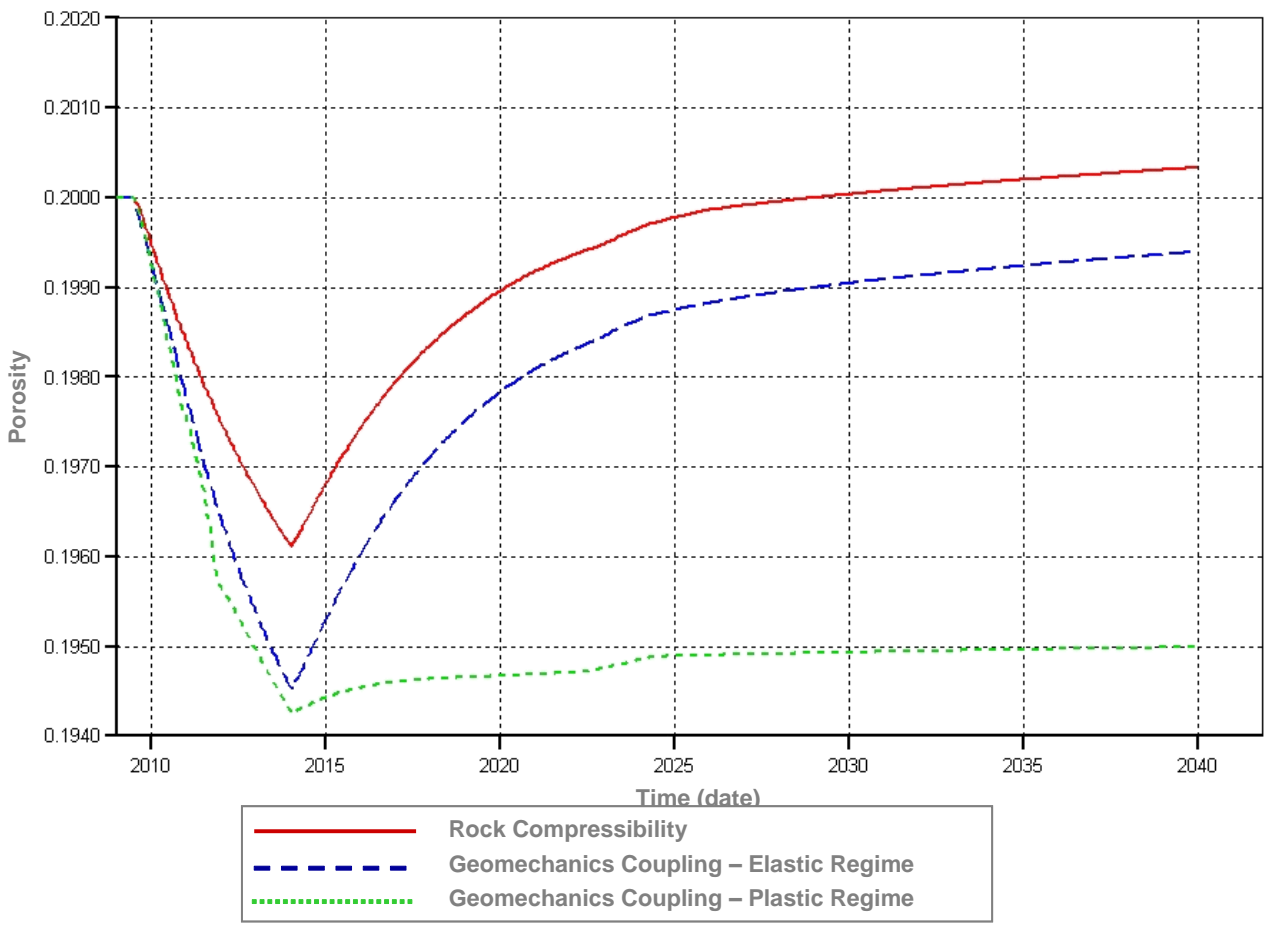

Figure 8 Porosity variation 


\section{Conclusions}

The results presented achieved the main objective of this work, which was to understand and analyse the geomechanical coupled influence on reservoir simulation. The understanding of this influence allows us to contribute to some important decisions, which range from water management aspects to production unit specification, based on oil production rate peak. By considering Figures 3 and 5 that identify the geomechanical parameters that really impact on the results, it is also possible to contribute to defining which geomechanical parameters should be obtained in a more accurate way.

The cap position was shown to be the most important parameter in the simulation, i.e. the knowledge of elastic or plastic behaviour of reservoir rock (collapse or non-collapse prediction), is the most important aspect on reservoir behaviour for this model. On the other hand, its variation cause problems on response surface generation, besides some numerical problems that negatively affect the simulation convergence.

Analysing the production curves presented in Figure 6, it shows an increase in cumulative oil production which leads us to conclude that compaction drive mechanism is acting. This interpretation is detailed in Figure 9, which presents the cumulative oil production curves and the respective variations in oil rate shown in both axes.

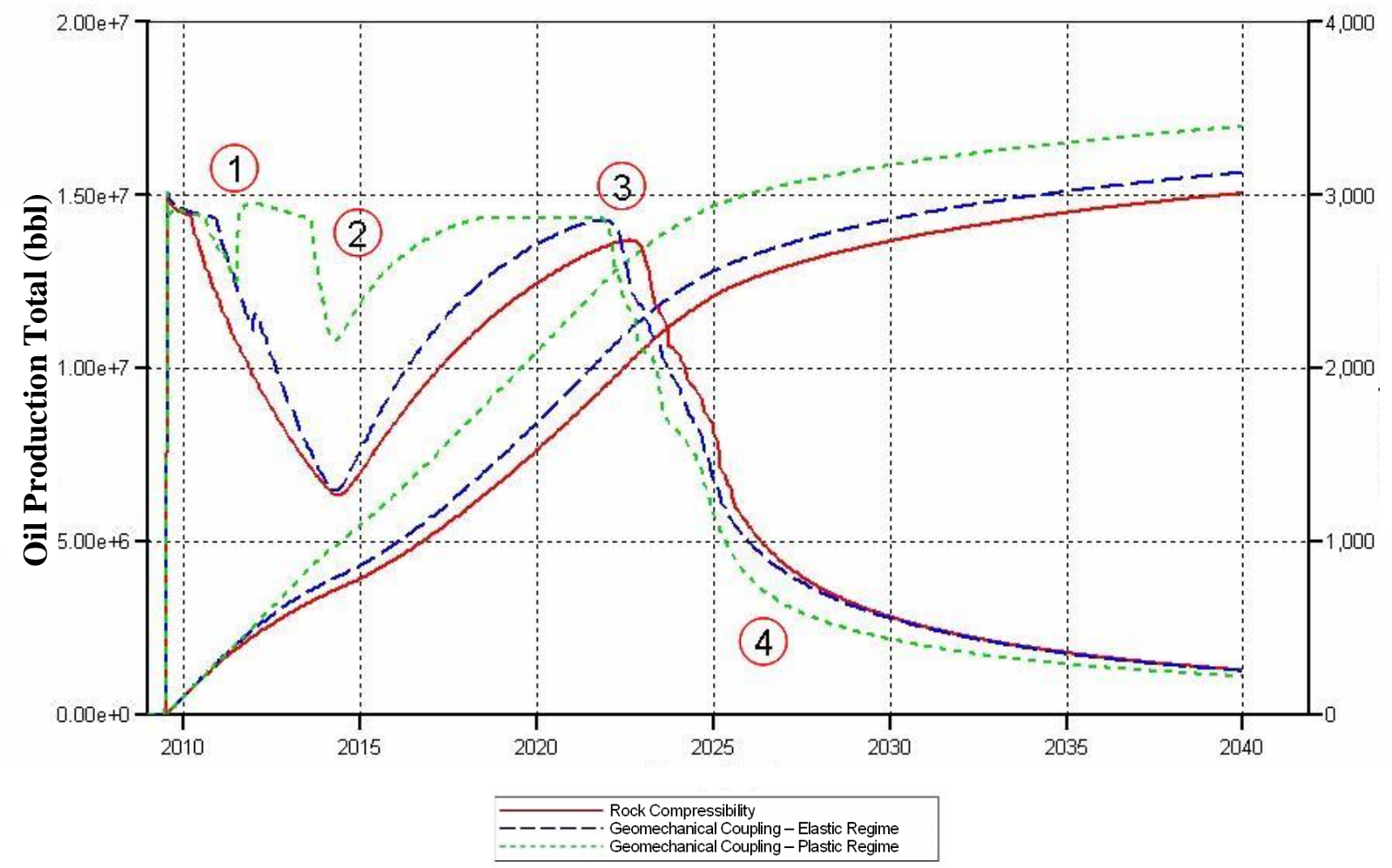

\section{Figure 9 Cumulative oil production and oil rate}

Figure 9 shows along with the cumulative oil production curves the respective oil rate curves for each case analysed. The full line present the conventional simulation results, while the dashed lines show the results of coupled simulation in the elastic and plastic regime, respectively. The numbered points indicate the instances where important events occurred.

Point 1 show the instant the reservoir rock reaches the plasticity surface. At this time the oil rate (dashed line) reaches a peak that may be explained by the excessive deformation deriving from the behaviour changing and consequent expulsion of fluids present in the pore volume. The difference between the conventional simulation and the coupled curves, still in the elastic regime, is due to deformation, with the elastic case having less intense deformation, responsible for compaction and consequent increase in the production rate. 
As described previously, the injector well is opened at 31 December 2013, exactly when the oil rate curve inclination changes abruptly. At point 2 there is a subtle difference between the oil rate curve inclinations. This difference is associated to the behaviour presented in Figure 8. The reservoir average porosity, even when the pressure is being reestablished, does not increase due to pore collapse occurrence. This phenomenon may cause serious well stability problems and substantially affects the recovery factor. Then, the pore collapse phenomenon may be irreversible and impair the field production. Still in Figure 8, there is a considerable difference on the average porosity variation, even within the elastic regime. The porosity and permeability variation in the reservoir due to production are important phenomena but are considered in an extremely simplified form in the conventional simulation.

Point 3 is associated with breakthrough. The difference of approximately 10 months is explained by Equation (7), which shows that the apparent velocity (water velocity) is directly associated to porosity and, consequently, the water arrives faster at the producer well when considering geomechanical modelling. Following the chart of Figure 9, point 4 shows the influence of permeability variations on the results. With the increase of effective stresses and consequent deformations, permeability decreases and could reduce the oil production rate.

In quantitative terms, when considering the geomechanical coupling, the oil production increase was $4.3 \%$ for elastic regime, and $13.2 \%$ considering that the plastic regime was achieved.

It must be emphasised that in this case there was an oil production gain because the effect of compaction drive mechanism exceeded the loss from the permeability variation, which is stress dependent. These phenomena are overlaid and the result, in some other situation, may be a production loss, depending on the reservoir characteristics and mainly on the magnitude of geomechanical parameters.

\section{References}

Biot, M.A. (1941) General Theory of Three-Dimensional Consolidation, Journal of Physics, Vol.12, pp. 155-164.

Chin, L.Y. and Boade, R.R. (1990) Full-Field, 3D Finite Element Subsidence Model for Ekofisk. 3rd North Sea Chalk Symposium, Copenhagen.

Fredrich, J.T., Arguello J.G., Thorne, B.J., Wawersik, W.R., Deitrick, G.L., Rouffignac, E.P., Myer, L.R. and Bruno, M.S. (1996) Three Dimensional Geomechanical Simulation of Reservoir Compaction and Implications for Well Failures in the Belridge Diatomite. SPE Annual Technical Conference and Exhibition. SPE 36698, Denver, Colorado, USA.

Fung, L.S.K., Buchanan, L. and Wan, L.G. (1994) Coupled Geomechanical-Thermal Simulation for Deforming HeavyOil Reservoir. J. Cdn. Pet. Tech., 22.

Gai, X., Dean, R.W., Wheeler, M.F. and Liu, R. (2003) Coupled Geomechanical and Reservoir Modeling on Parallel Computers. SPE Reservoir Simulation Symposium, SPE 79700, Houston, Texas, USA.

Gutierrez, M. and Lewis, R.W. (1998) The Role of Geomechanics in reservoir simulation. In: SPE/ISRM Eurock'98, pp. 439-448, Norway.

Hart, R.D. and John, C.M.St. (1986) Formulation of a Fully-Coupled Thermal-Mechanical Fluid Flow Model for NonLinear Geologic Systems. International Journal Rock Mechanics Min. Sci. and Abstr. Geomech., Vol. 03, pp. 213-224.

Koutsabeloulis, N.C. and Hope, S.A. (1998) Coupled Stress/Fluid/Thermal Multi-phase Reservoir Simulation Studies Incorporating Rock Mechanics. In: SPE/ISRM Eurock'98, SPE 47393, Norway.

Lewis, R.W. and Sukirman, Y. (1993) Finite Element Modeling of Three-phase Flow in Deforming Saturated Oil Reservoirs. Intl. J.for Num. and Anal. Methods in Geomech., 17, p. 577.

Lin, C.C. and Segel, L.A. (1974) Mathematics Applied to Deterministic Problems in the Natural Sciences. 1st edition, New York, MacMillan.

Minkoff, S.E., Stone, C.M., Arguello, J.G., Bryant, S., Eaton, J., Peszynska, M. and Wheeler, M. (1999) Staggered in Time Coupling of Reservoir Flow Simulation and Geomechanical Deformation: Step 1 - One-way Coupling. SPE Reservoir Simulation Symposium, SPE 51920, Houston, Texas, USA.

Osorio, J.G., Chen, H.Y., Teufel, L.W. and Schaffer, S. (1998) A Two-Domain, 3D, Fully Coupled FluidFlow/Geomechanical Simulation Model for Reservoirs with Stress-Sensitive Mechanical and Fluid-Flow Properties. In: SPE/ISRM Eurock'98, Norway, pp. 455-464.

Osorio, J.G., Chen, H.Y. and Teufel, L.W. (1999) Numerical Simulation of the Impact of Flow Induced Geomechanical Response on the Productivity of Stress-Sensitive Reservoirs. SPE Reservoir Simulation Symposium, SPE 51929, Houston, Texas, USA.

Settari, A. and Walters, D.A. (1999) Advances in Coupled Geomechanics and Reservoir Modeling with Applications to Reservoir Compaction. SPE Reservoir Simulation Symposium, SPE 51927, Houston, Texas, USA. 
Stars Technical Guide (2001) Computer Modelling Group Ltd.

Sulak, R.R., Thomas, L.K., and Boade, R.R. (1991) 3D Reservoir Simulation of Ekofisk Compaction Drive, Journal of Petroleum Technology pp. 1272-1278.

Terzaghi, K. (1943) Theoretical Soil Mechanics. 1st edition, New York, John Wiley \& Sons.

Tortike, W.S. and Ali, F. (1992) Reservoir Simulation Integrated with Geomechanics. Proceedings CIM Annual Technical Conference, Calgary, Canada. 